\title{
FEATURES EXTRACTION OF THE DOPPLER FREQUENCY SIGNATURE OF A HUMAN WALKING AT 1 GHz.
}

\author{
Giovanni Manfredi ${ }^{*, \dagger}$, Jean-Philippe Ovarlez ${ }^{*, \dagger}$ and Laetitia Thirion-Lefevre ${ }^{\dagger}$ \\ ^DEMR, ONERA, Université Paris-Saclay, F-91123 Palaiseau, France \\ ${ }^{\dagger}$ SONDRA, CentraleSupélec, Université Paris-Saclay, F-91190, Gif-sur- Yvette, France
}

\begin{abstract}
A preliminary Doppler analysis in the UHF-band on a man walking in free space is presented in this paper. Firstly, the back-scattered response of the moving target has been provided at $1 \mathrm{GHz}$ by a simulation tool based on physical optics theory. Then, a short-time Fourier transform (STFT), a reassignment spectrogram (RE-Spect) and a Wigner-Ville distribution (WVD) have been employed to the data, in order to characterize the micro-Doppler signature of the human body walking. The results are based entirely on numerical tests. The investigation on the time variations of the Doppler spectrum of moving targets in UHF-band is of interest for the emerging radar applications devoted to the detection and tracking of people in highly cluttered environment.
\end{abstract}

Index Terms - Feature extraction, micro-Doppler, timefrequency analysis, spectrogram

\section{INTRODUCTION}

The growing demand for physical security and surveillance has renewed a significant interest on radar applications, such as sensing through the wall (STTW) and foliage penetration (FOPEN) radar, addressed to detect and track people in cluttered environment. The major challenge is detect and classify a moving human from any moving objects (e.g. leaves or animals present in the scene) which produce a Doppler response, thereby creating false alarms.

The Doppler human return is characterized by the appearance of micro-Doppler features [1,2], generated from non rigid body motions, making the signature of human movement unique. There has been a significant amount of research focused on the Doppler human spectrum. For instance, van Dorp [3] estimated the parameters of human gait from frequency modulated continuous wave radar data. In [4] a time-frequency analysis is carried out to distinguish the backscattered response of a human by that of a quadruped. Kim [5] provided a classification based on micro-Doppler features of the various human activities such as walking, running, crawling, etc., using a support vector machine. Du [6] proposes a novel feature extraction method based on micro-Doppler signature, to distinguish single walking person from two peo- ple walking or moving wheeled vehicle. These studies have shown promising results in X-band and K-band. Actually, the Doppler signature of human motions has to be investigated in the UHF-band, which is of interest for radar applications involved on FOPEN detection context. The research presented in this paper represents a preliminary study in this direction.

In detail, the Doppler spectrum of a human walking in free space has been analyzed. The moving human body has been modelled by canonical geometric shapes, whose backscattering response has been provided at $1 \mathrm{GHz}$, by a simulation tool based on physical optics theory. Five features have been extracted from the spectrograms once data have been post-processed by using a STFT, a RE-Spect and a WVD, and compared with the analytical results. The purpose is to highlight what time-frequency analysis allows the best characterization of the micro-Doppler signature of an human physical activity in UHF-band.

The paper is organized as follows. The simulation setup, the analytical results and the methodology involved for the extraction of the Doppler features are described in Section II. The spectrograms of the monitored moving target are compared and discussed in Section III. In Section IV, the conclusions are presented.

\section{DOPPLER FEATURES OF A MAN WALKING AT 1 GHz}

\subsection{Computer model}

The Doppler analysis focused on a real subject walking in free space toward an antenna. The motion trajectories of the target have been acquired by Microsoft Kinect v2, and translated in relative angles and distances [7]. Once acquired the body shapes and gestures of the subject walking, an equivalent 3D human model has been implemented in MATLAB ${ }^{\circledR}$, replacing each body parts with canonical dielectric shapes (sphere, cylinders and parallelepiped). The dielectric properties $\varepsilon_{r}=50$ and $\sigma=1 \mathrm{~S} / \mathrm{m}$ have been chosen to be close to the skin properties [8].

The electromagnetic (EM) signal has been generated by a horn antenna, placed at $8 \mathrm{~m}$ away the target and operating in continuous wave at $1 \mathrm{GHz}$, with a gain of $10 \mathrm{~dB}$ and a radi- 
ated power of $0 \mathrm{dBm}$. Vertical-Vertical polarization $(\mathrm{V}-\mathrm{V})$ has been considered for the numerical tests. The total reflected EM field has been provided in frequency domain by an analytical model based on physical optics (PO) theory.

A total observation time of $8 \mathrm{~s}$ has been analyzed during which the walking cycle completes in $2 \mathrm{~s}$, with an averaged velocity of $0.4 \mathrm{~m} / \mathrm{s}$. In order to extract the Doppler shifts, the walking motion has been decomposed into 241 frames that succeed each other with a pulse repetition frequency (PRF) of $30 \mathrm{~Hz}$. The PRF has been chosen to avoid aliasing in the Doppler domain [9] at the highest radar frequency detected in this study.

The generation of the human model, the characterization of the scattered fields and the signal processing routines have been implemented in MATLAB ${ }^{\circledR}$.

\subsection{Analytical Doppler frequencies of the human body elements}

The human model has been modelled as having 11 body parts in order to reproduce faithfully the motions of a human walking cycle: head $(\mathrm{H})$, neck $(\mathrm{N})$, torso $(\mathrm{T})$, right arm (RUA), left arm (LUA), right forearm (RLA), left forearm (LLA), right thigh (RUL), left thigh (LUL), right leg (RLL) and left leg (LLL). The Doppler frequencies of each body part have been analytically calculated to highlight individually their contribution, by knowing their positions during the walking motion. The graph of the Doppler frequencies is shown in Fig. 1.

As already presented in [5] by Y. Kim and H. Ling, five features have been chosen to characterize the Doppler and micro-Doppler shifts of the physical activity at $1 \mathrm{GHz}$ : (1) the torso Doppler frequency, (2) the total bandwidth (BW) of the Doppler signal, (3) the offset, (4) the BW without microDoppler, and (5) the period. The torso Doppler frequency (1) is related to the speed of the human target whose variation depends on the different physical activities. The total BW of the Doppler signal (2) corresponds to the speed of the upper and

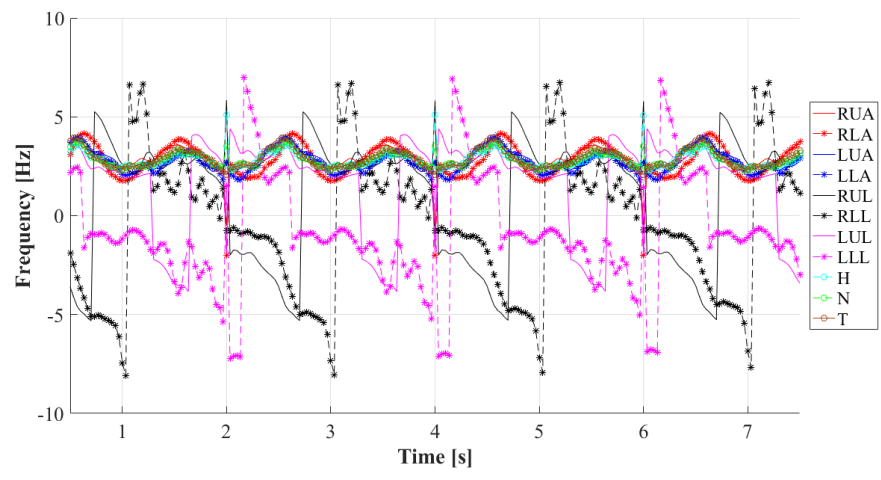

Fig. 1: Doppler frequencies of human body walking directly towards the radar at $1 \mathrm{GHz}$.
Table 1: Doppler features of a man walking analytically calculated at $1 \mathrm{GHz}$

\begin{tabular}{|l|c|}
\hline Features & Values \\
\hline$(1)$ & $2.86 \mathrm{~Hz}$ \\
$(2)$ & $15.08 \mathrm{~Hz}$ \\
$(3)$ & $0.56 \mathrm{~Hz}$ \\
$(4)$ & $3.16 \mathrm{~Hz}$ \\
$(5)$ & $2 \mathrm{~s}$ \\
\hline
\end{tabular}

lower limbs. The offset (3) outlines the asymmetry between the forward and backward movements of the limbs. The BW without micro-Doppler (4) corresponds to the Doppler BW of the torso alone. The period (5) represents the swing rate of the limbs.

The five features have been automatically extracted by processing the data over each time bin. In detail, for the calculation of (2), (3), and (4), two envelopes are identified: the high-frequency envelope (HFE) and the low-frequency envelope (LFE). At each time bin, HFE is made up by the highest Doppler frequency, whereas LFE is made up by the lowest Doppler frequency. It follows that, the BW (2) is the averaged difference between the highest frequency of HFE and the lowest frequency of LFE. The offset (3) is the mean value between the largest frequency of HFE and the smallest of LFE. The BW without micro-Doppler (4) is the averaged difference between the lowest frequency from HFE and the highest from LFE. The values of the Doppler features related to the analytical curves are listed in Table 1. The torso Doppler frequency $(1)=2.86 \mathrm{~Hz}$ means an human subject walking with an averaged speed around $0.43 \mathrm{~m} / \mathrm{s}$ during the observation time. The swinging of the lower limbs entails a maximum Doppler shift (2) of $15 \mathrm{~Hz}$, with quite symmetric forward and backward motions as highlighted by the offset value (3) close to 0 . The BW without the micro-Doppler (4) results higher than (1) due to the bobbing motions of the upper limbs. The period of the monitored physical activity corresponds to the walking cycle which ends every $2 \mathrm{~s}$.

\section{TIME-FREQUENCY ANALYSIS OF A MAN WALKING}

The back-scattered signal provided by the EM solver has been firstly frequency modulated with an intermediate frequency $f_{I F}=4.75 \mathrm{~Hz}$. Then, the modulated signal has been postprocessed performing a STFT [10], a RE-Spect [11,12] and a WVD [13], in order to extract the five Doppler features. They represent a class of window-based transformation functions which allow to analyze the time variation of the Doppler spectrum of a moving target. Hanning, Hamming, Gauss, Kaiser and Flapttop have been chosen as frequency windows for the Doppler analysis, with a coherent processing interval (CPI) equal $1.3 \mathrm{~s}$, which implies a frequency resolution $\Delta f$ of 0.77 


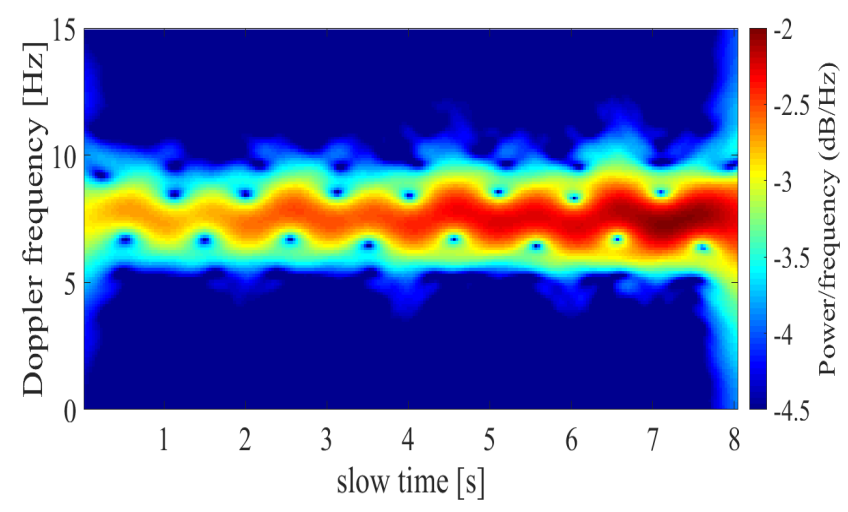

Fig. 2: STFT: Doppler spectrogram of a man walking.

Table 2: Short-time Fourier transform: Doppler Features

\begin{tabular}{|l|c|c|c|c|c|}
\hline Features & Hanning & Hamming & Gauss & Kaiser & Flapttop \\
\hline$(1)$ & $2.84 \mathrm{~Hz}$ & $2.84 \mathrm{~Hz}$ & $2.84 \mathrm{~Hz}$ & $2.83 \mathrm{~Hz}$ & $2.81 \mathrm{~Hz}$ \\
$(2)$ & $9.02 \mathrm{~Hz}$ & $9.5 \mathrm{~Hz}$ & $9.5 \mathrm{~Hz}$ & $9.37 \mathrm{~Hz}$ & $10.4 \mathrm{~Hz}$ \\
$(3)$ & $3.21 \mathrm{~Hz}$ & $3.21 \mathrm{~Hz}$ & $3.21 \mathrm{~Hz}$ & $3.26 \mathrm{~Hz}$ & $3.21 \mathrm{~Hz}$ \\
$(4)$ & $4.10 \mathrm{~Hz}$ & $3.98 \mathrm{~Hz}$ & $3.75 \mathrm{~Hz}$ & $4.33 \mathrm{~Hz}$ & $5.03 \mathrm{~Hz}$ \\
$(5)$ & $1.97 \mathrm{~s}$ & $1.97 \mathrm{~s}$ & $2 \mathrm{~s}$ & $1.97 \mathrm{~s}$ & $2.1 \mathrm{~s}$ \\
\hline
\end{tabular}

$\mathrm{Hz}$. The results are plotted in $\mathrm{dB} / \mathrm{Hz}$ scale, where the abscissa represents the slow time, whereas the ordinate the Doppler frequency shift. The spectrograms presented in this section are related to the employing of the Hanning window. The graphs represent four walking cycles, in which the data generated by the first cycle is simply repeated another three times.

The first analysis focused on the STFT of the back-scattering response of the target walking straight toward the antenna. The resulting spectrogram is shown in Fig. 2, whereas the relative Doppler features are listed in Table 2, to which values the $f_{I F}$ was subtracted. The physical activity is well detectable and some Doppler features, mentioned in the previous section, can be characterized. The torso Doppler frequency (1), is represented in Fig. 2 by the strongest returned signal in the middle of the diagram. Table 2 shows that (1) varies from $2.81 \mathrm{~Hz}$ and $2.84 \mathrm{~Hz}$ which relative speed is closely to the speed of the human subject of about $0.43 \mathrm{~m} / \mathrm{s}$. The BW of the total Doppler (2) is related to the legs motions which exhibit highest Doppler shift around $9 \mathrm{~Hz}-10.4 \mathrm{~Hz}$, but weaker in magnitude. The period (5) of about $2 \mathrm{~s}$ is mainly highlighted at $2 \mathrm{~s}, 4 \mathrm{~s}$ and $6 \mathrm{~s}$ by the spurious jumps at the beginning and end of each walking cycle.

Comparing the simulated results shown in the Table 2 with those listed in Table 1, a good accuracy was achieved for the features (1) and (5). Conversely, the extraction of (2), (3) and (4) proves to be unreasonable. The error is caused by the low chosen working frequency which implies lower frequency shifts. It follows that, a poor frequency resolution doesn't allow to correctly identify the high and low frequency envelopes, and as consequence, to faithfully characterize the

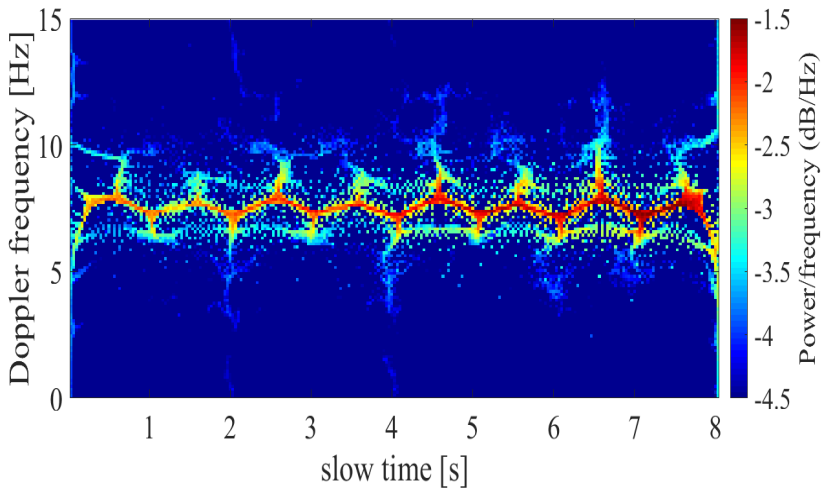

Fig. 3: RE-Spect: Doppler spectrogram of man walking.

Table 3: Reassignment spectrogram: Doppler Features

\begin{tabular}{|l|c|c|c|c|c|}
\hline Features & Hanning & Hamming & Gauss & Kaiser & Flapttop \\
\hline$(1)$ & $2.81 \mathrm{~Hz}$ & $2.81 \mathrm{~Hz}$ & $2.81 \mathrm{~Hz}$ & $2.81 \mathrm{~Hz}$ & $2.83 \mathrm{~Hz}$ \\
$(2)$ & $14.88 \mathrm{~Hz}$ & $12.53 \mathrm{~Hz}$ & $14.88 \mathrm{~Hz}$ & $14.88 \mathrm{~Hz}$ & $14.29 \mathrm{~Hz}$ \\
$(3)$ & $2.74 \mathrm{~Hz}$ & $2.97 \mathrm{~Hz}$ & $2.74 \mathrm{~Hz}$ & $2.74 \mathrm{~Hz}$ & $2.56 \mathrm{~Hz}$ \\
$(4)$ & $0.94 \mathrm{~Hz}$ & $0.82 \mathrm{~Hz}$ & $0.58 \mathrm{~Hz}$ & $0.82 \mathrm{~Hz}$ & $0 \mathrm{~Hz}$ \\
$(5)$ & $2 \mathrm{~s}$ & $2.03 \mathrm{~s}$ & $2 \mathrm{~s}$ & $2 \mathrm{~s}$ & $2.1 \mathrm{~s}$ \\
\hline
\end{tabular}

main Doppler features.

The second analysis focused on the application of RESpect to the same data. The corresponding spectrogram is shown in Fig. 3 and the relative Doppler features are reported in Table 3. The employ of the RE-Spect improved the readability of the time frequency representation shown Fig. 3. The Doppler frequency (1) as well as the total BW (2) and the period of the movement (5) are well detectable and in accordance with those listed in Table 1. Whereas the MATLAB routine process makes still a mistake when evaluating the offset (3) and the BW without micro-Doppler (4).

In the end, a pseudo WVD has been carried out to the back-scattered data. The spectrogram is shown in Fig. 4 and the results presented in Table 4 . The Doppler spectrum of

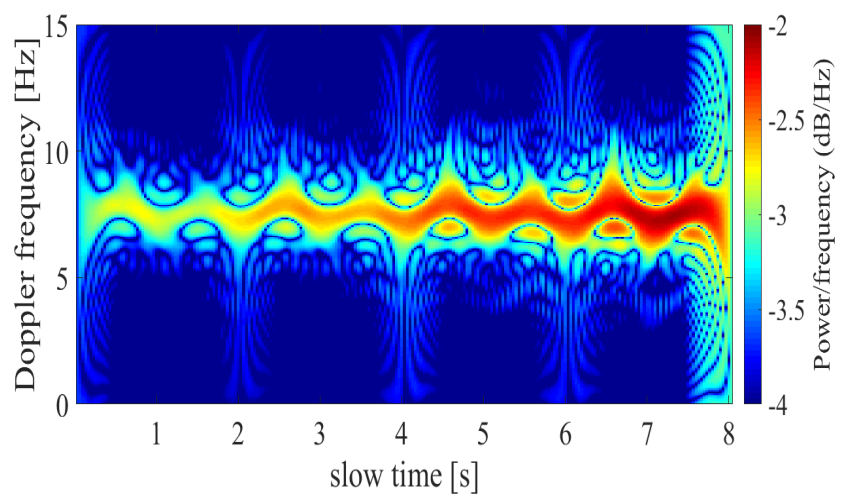

Fig. 4: WVD: Doppler spectrogram of man walking. 
Table 4: Wigner-Ville distribution: Doppler Features

\begin{tabular}{|l|c|c|c|c|c|}
\hline Features & Hanning & Hamming & Gauss & Kaiser & Flapttop \\
\hline$(1)$ & $2.81 \mathrm{~Hz}$ & $2.81 \mathrm{~Hz}$ & $2.82 \mathrm{~Hz}$ & $2.82 \mathrm{~Hz}$ & $2.82 \mathrm{~Hz}$ \\
$(2)$ & $14.94 \mathrm{~Hz}$ & $14.94 \mathrm{~Hz}$ & $14.94 \mathrm{~Hz}$ & $14.94 \mathrm{~Hz}$ & $14.94 \mathrm{~Hz}$ \\
$(3)$ & $2.77 \mathrm{~Hz}$ & $2.77 \mathrm{~Hz}$ & $2.77 \mathrm{~Hz}$ & $2.77 \mathrm{~Hz}$ & $2.77 \mathrm{~Hz}$ \\
$(4)$ & $3.34 \mathrm{~Hz}$ & $3.46 \mathrm{~Hz}$ & $2.64 \mathrm{~Hz}$ & $2.64 \mathrm{~Hz}$ & $3.05 \mathrm{~Hz}$ \\
$(5)$ & $1.93 \mathrm{~s}$ & $1.93 \mathrm{~s}$ & $1.93 \mathrm{~s}$ & $1.93 \mathrm{~s}$ & $1.93 \mathrm{~s}$ \\
\hline
\end{tabular}

the human body walking is well outlined, even though it is affected by the interference terms (due to the bi-linearity of the WVD), observed on the top and the bottom of Doppler sinusoidal envelope. Table 4 highlights that a good accuracy can be achieved for only four of the main Doppler features. Whereas the offset (3) proves to be not in accordance with the expected result as already observed in the previous analysis. The issues is that the offset represents the asymmetry of the forward and backward motions of the limbs which exhibit the highest Doppler shift but undetectable by the our process, being weaker in magnitude. The Doppler features extraction is preceded by a processing of the returned signal with a threshold equal to $-4.5 \mathrm{~dB} / \mathrm{Hz}$, chosen in order to avoid the spurious terms which affect the readability of the spectrograms.

\section{CONCLUSION}

In this paper we investigated the feasibility to analyze at $1 \mathrm{GHz}$ the Doppler spectrum of a man walking. The numerical tests have been carried out analyzing firstly the backscattering response of the moving target, and then employing three different joint time-frequency techniques. Five main Doppler features have been extracted both to characterize the frequency signature of moving subject and to discriminate the best timefrequency analysis. Despite the chosen low frequency, the walking was well detectable and the pseudo Wigner-Ville distribution proved to be the best time-frequency function to detect and characterize the physical activity of a real subject.

Currently, outdoor experimental tests are ongoing at ONERA, observing man walking and running bordering and within the forest. The aim is to provide a feasibility study of the Doppler analysis in the UHF-band and useful information relative the time-frequency distribution of people moving in complex cluttered environment, for FOPEN applications.

\section{REFERENCES}

[1] V. C. Chen, F. Li, S. S. Ho, and H. Wechsler, "MicroDoppler effect in Radar: phenomenon model and simulation study," IEEE Transactions on Aerospace and electronic systems, vol. 42, no. 1, pp. 2-21, 2006.

[2] V. C. Chen and H. Ling, Time-Frequency transforms for radar imaging and signal analysis, Artech House, 2002.
[3] P. van Dorp and F. C. A. Groen, "Human walking estimation with radar," IEE Proceedings-Radar, Sonar and Navigation, vol. 150, no. 5, pp. 356-365, 2003.

[4] M. Vahidpour and K. Sarabandi, "Millimeter-wave Doppler spectrum and polarimetric response of walking bodies," IEEE Transactions on Geoscience and Remote Sensing, vol. 50, no. 7, pp. 2866-2879, 2012.

[5] Y. Kim and H. Ling, "Human activity classification based on micro-Doppler signatures using a support vector machine," IEEE Transactions on Geoscience and Remote Sensing, vol. 47, no. 5, pp. 1328-1337, 2009.

[6] L. Du, L. Li, B. Wang, and J. Xiao, "Micro-Doppler feature extraction based on time-frequency spectrogram for ground moving targets classification with low-resolution radar," IEEE Sensors Journal, vol. 16, no. 10, pp. 37563763, 2016.

[7] M. Capecci, M. G. Ceravolo, F. Ferracuti, S. Iarlori, V. Kyrki, S. Longhi, L. Romeo, and F. Verdini, "Physical rehabilitation exercises assessment based on hidden semi-markov model by Kinect v2," in Biomedical and Health Informatics (BHI), 2016 IEEE-EMBS International Conference on. IEEE, Feb 2016, pp. 256-259.

[8] T. Dogaru, C. Le, and G. Kirose, "Time-frequency analysis of a moving human Doppler signature," Tech. Rep., Army Research Lab Adelphi MD Sensors and Electron Devices Directorate, 2009.

[9] M. I. Skolnik, "Introduction to radar systems, mcgrawhill, comp," New York, 2001.

[10] V. C. Chen and S. Qian, "Joint Time-Frequency transform for Radar range-Doppler imaging," IEEE Transactions on Aerospace and Electronic Systems, vol. 34, no. 2, pp. 486-499, 1998.

[11] F. Auger and P. Flandrin, "Improving the readability of Time-Frequency and time-scale representations by the reassignment method," IEEE Transactions on Signal Processing, vol. 43, no. 5, pp. 1068-1089, 1995.

[12] I. Reinhold, J. Starkhammar, and M. Sandsten, "The scaled reassigned spectrogram adapted for detection and localisation of transient signals," in Signal Processing Conference (EUSIPCO), 2017 25th European. IEEE, 2017, pp. 907-911.

[13] R. Tan, H. S. Lim, A. B. Smits, R. I. A. Harmanny, and L. Cifola, "Improved micro-Doppler features extraction using Smoothed-Pseudo Wigner-Ville distribution," in Region 10 Conference (TENCON), 2016 IEEE. IEEE, 2016, pp. 730-733. 